\title{
Efficacy of Oral Mini Pulse Betamethasone in the Treatment of Vitiligo
}

\author{
L Khondker, ${ }^{1}$ M S I Khan, ${ }^{2}$ S C Hazra, ${ }^{3}$ N Ahmed, ${ }^{4}$
}

\begin{abstract}
Vitiligo is a multifactorial polygenic disorder with a complex pathogenesis, characterized by depigmented macules in skin. A clinical trial was conducted to evaluate the efficacy of oral mini pluse betamethasone in the treatment of vitiligo patients. The study was done in the department of Dermatology and Venerology, Bangabandhu Sheikh Mujjb Medical University, Dhaka, Bangladesh for duration of July 2009 to June 2010. Among thirty patients of vitiligo, most of the male patients (60\%) and most of the femal patients (73\%) were between 20 to 25 years. Majority of patients $(70 \%)$ had been suffering from vitiligo for less than 3 years and $53 \%$ patients had family history.
\end{abstract}

Key words: Efficacy of betamethasone, side-effects of betamethasone, treatment of vitiligo.

\section{Introduction}

Vitiligo is an acquired pigmentary anomaly of the skin manifested by depigmented white patches surrounded by a normal or a hyperigmented border. ${ }^{1}$ The cause is unkown but may involve genetic factors, autoimmunity, toxic metabolites and/or a higher vulnerability of melanocytes. ${ }^{2-4}$ Vitiligo is associated with a number of autommine discorders, most common of which is hypothyroidism. ${ }^{5}$ Since curative treatment is not available, current modalities are directed towards stopping progression and to achieving regimentation in order to repair the morphology and functional deficiencies of depigmented skin areas. ${ }^{1,3,5}$ For widespread disease, photo chemotherapy is generally considered the first line treatment. ${ }^{4,5}$ The other modes of treatment include topical and systemic steroids, broad and narrow spectrum Ultraviolet $\mathrm{B}(\mathrm{UVB})$ and grafting techniques; minigrafts and autologous cultured melanocytes. $^{1,6}$

One of the most probable pathogeneses of vitiligo is autoimmunity. Systemic corticosteroids suppress immunity and may arrest the progression of vitligo and lead to regimentation. Systemic steroids may also prduce unaccepatable side effects. ${ }^{5,7}$ To minimize the side effects, we tried a new approach using oral mini pulse betamethasone in vitiligo patients.

1. Dr. Lubna Khondker, Assistant Professor, Dept of Dermatology and Venereology, BSMMU.

2. Major (Dr) Md Shirajul Islam Khan, Dermatology, Combined Military hospital (CMH), Dhaka cantonment.

3. Dr. Samaresh Chandra Hazra, Specialist in Dermatology and Venereology, BSMMU.

4. Dr. Nafiza Ahmed, Assistant Professor, Dept.of Dermatology, Dhaka Medical college Hospital.

\section{Corresponding Author}

Dr. Lubna Khondker, Assistant Professor, Dept of Dermatology and Venereology,BSMMU.
Twenty one (70\%) patients had acro-facial type vitiligo and 23(76.7\%) cases were progressive in nature. In this study, response was slight in $33.33 \%$, moderate in $26.67 \%$ marked in $33.33 \%$ and excellent in $6.67 \%$ of cases after 12 weeks. Twenty percent (20\%) of total patients were seen to be with clinical side effect. Among the side effects, weight gain 2(6.67\%), and acene 2(2.67\%) was most prevalent, others include general weakness 1(3.33\%) and mild headache 1(3.33\%). In conclusion, oral mini pulse betamethasone seems to be an effective treatment modality to arrest the progression of vitiligo and induction of repigmentation.

\section{Materials and Methods}

A clinical trial was carried out to assess the efficacy of oral mini pulse betamethasone in patients of vitiligo. The study was conducted in the department of Dermatology and Venereology, Bangabandhu Sheikh Mujjib Medical University, Dhaka, Bangladesh for duration of July 2009 to June 2010. The patients were given $5 \mathrm{mg}$ betamethasone as a single oral dose afer breakfast on 2 consecutive days per week for 16 weeks. The effects of treatment were evaluated using photographs of before and after the study. Side-effects were assessed at the first, second and third month of treatment. A total of thirty patients who fulfilled the inclusion criteria were enrolled in the study on an out patient basis after taking an informed consent. First 30 patients of either sex having vitiligo of more than six months duration whether stable of progressive reporting to Dermatology department were enrolled. Age of the patients was between 20 and 55 years. Patient with pregnancy, lactating mothers, patients receiving any treatment or those who had received any treatment during last 3 month which might influence the course f disease, including photo chemotherapy, Ultraviolet B Phototherapy, systemic illnesses like diabetes mellitus, hypertension, ischaemic heat disease, thyroid disorders or any other systemic autoimmune disorder were also excluded from the study. Detailed history was taken and physical examination was performed. Type of vitiligo whether generalized, acrofacial or focal was determined by history and physical examination. Extent of vitiligo was assessed by lesion counting and determination of percentage of body surface area affected by rule of nine. Course of disease was determined by history. The disease was considered stable if no new lesion had appeared and preexisting lesion had not enlarged during last six months. Baseline laboratory investigations that were carried out for purpose of exclusion and monitoring of side effects included, complete blood counts, serum urea, serum creatinine and electrolytes, plasma glucose fasting and 2 hours after breakfast, liver function tests and serum cortisol levels. All the patients were weighed before starting of treatment. Photographic documentation with close up photographs of all the depigmented macules was done in all patients after getting ingormed consent from the patient. In the first and fourth week of therapy plasma cortisol levels were determined in all patients 
before the first dose (day 0 ) as well as 48 hours after, before 4th dose and 48 hers after. The clinical respons was evaluated at monthly intervals. Percentage of repigmentation was estimated on each visit by lesion counting and a visual comparison of the patients against their baseline phothographs. Clinical evaluation was done at baseline and the end of the treatment. In addition to the treatment response, type and frequency of side effects were recorded at each visit by taking history of general weakness, weight gain, mild headache, acne, epigastric pain and menstrual abnormality in case of female. Patients were weighed at each visit to detect any weight gain. At the end of study, complete blood counts, serum urea, serum creatinine and electrolytes, blood glucose fasting and 2 hours after breakfast and liver function tests were preformed in all patients. Efficacy parameters included arrest of progression and percentage of regimentation. Progression was said to be arrested if no lesion appeared or pre-existing lesions did not enlarge during last one month. Lesion counting and a visual comparison of the patients against their baseline photographs estimated the percentage of repigmentation. Repigmentaton was considered excellent $(>75 \%)$, marked (51\% to $75 \%$ ), moderate (26\% to $50 \%$ ) and slight (25\% or less). Safety of treatment was assessed by incidence of side effects as judged by history, clinical examination and laboratory investigations.

\section{Results}

Among thirty patients of vitiligo, most of the male patients $(60 \%)$ and most of the female patients (73\%) were between 20 to 25 years. Majority of patients $(70 \%)$ had been suffering from vitiligo for less than 3 years and $53 \%$ patients had family history of vitiligo. Twenty one (70\%) patients had acro-facial and nine patients (30\%) had generalized vitiligo. The disease was stable in $7(23.3 \%)$ and progressive in $23(76.7 \%)$ cases. The extent of cutaneous involvement was less than $30 \%$ area in $21(70 \%)$ patients, 31 to $50 \%$ area in $8(27 \%)$ patients and more than $50 \%$ area in $1(3 \%)$ patients. In this study response was noticed at first follow-up after 4 weeks, then after weeks and 12 weeks. Response was slight in $73.33 \%$ moderate in $20 \%$, marked in $3.33 \%$ and excellent in $3.33 \%$ of cases after 4 weeks. Response was slight in $50 \%$, moderate in $40 \%$, marked in $6.67 \%$ and excellent in $3.33 \%$ of cases after 8 weeks. Response was slight in $33.33 \%$, moderate in $26.67 \%$, marked in $33.33 \%$ and excellent in $6.67 \%$ of cases after 12 weeks. In the present study $20 \%$ of total patients were seen to be with clinical side effect. Among the side effects weight gain (6.67\%), and acne $(6.67 \%)$ was most prevalent, others include general weakness $(3.33 \%)$ and mild headache $(3.33 \%)$. Plasma cortisol levels were markedly decreased 48 hours after and the second oral mini pulse betamethasone. However, betamethasone induced suppression of endogenous cortisol production was only transitory since plasma cortisol value returned to baseline levels before administration of the next oral mini pulse betamethasone therapy.
Table 1: Characteristics of patients of vitiligo.

\begin{tabular}{|lcccc|}
\hline Age in years & \multicolumn{3}{c}{ Male } & \multicolumn{3}{c|}{ Female } \\
& No. & Percentage No. & Percentage \\
$15-20$ & 4 & 27 & 3 & 20 \\
$20-25$ & 9 & 60 & 11 & 73 \\
$25-30$ & 1 & 7 & 1 & 7 \\
$30-35$ & 1 & 7 & 0 & 0 \\
Duration in year & & Number & & Percentage \\
$<3$ & & 21 & & 70 \\
$3-6$ & & 8 & & 27 \\
6-10 & & 1 & & 3 \\
Family History & & Number & Percentage \\
Positive & & 16 & & 53 \\
Nagative & & 14 & & \\
\hline
\end{tabular}

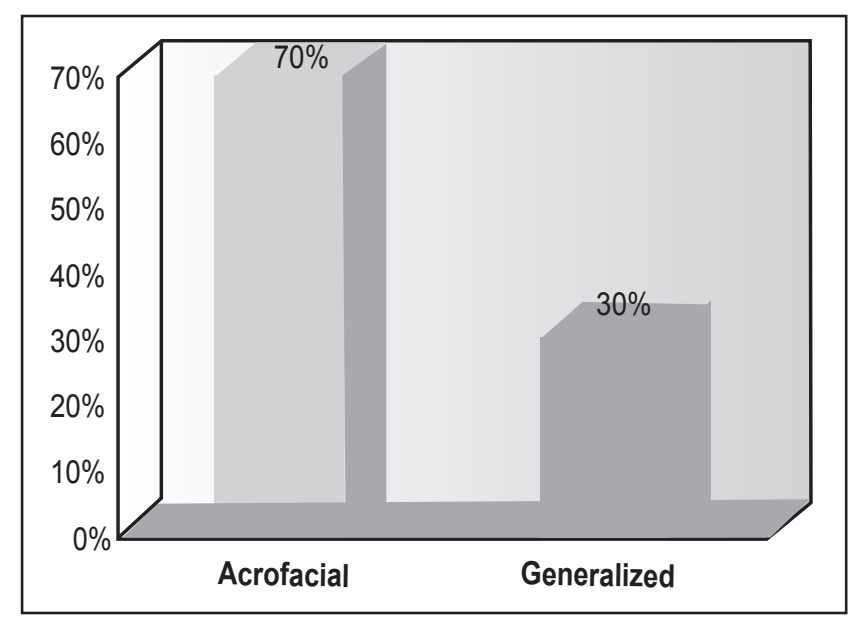

Figure 1: Distribution of the patients by varieties of vitiligo.

Table II. Distribution of patients by status extent of vitligo $(n=30)$

\begin{tabular}{|lcc|}
\hline Status of vitiligo & Number & Percentage \\
Stable & 7 & $23.3 \%$ \\
Progressive & 23 & $76.7 \%$ \\
Extent of involvement & Number & Percentage \\
$<30 \%$ & 21 & 70 \\
$31-50 \%$ & 8 & 27 \\
$>50 \%$ & 1 & 3 \\
\hline
\end{tabular}


Table III. Distribution of response rate according to duration $(n=30)$

\begin{tabular}{|lcrrrr|}
\hline Efficacy & \multicolumn{2}{c}{ Week- 4} & \multicolumn{2}{c}{ Week- 8} & Week- 12 \\
No. & Percentage No. & Percentage No & Percentage \\
Excellent1 & 3.33 & 1 & 3.33 & 2 & 6.67 \\
Marked 1 & 3.33 & 2 & 6.67 & 10 & 33.3 \\
Maderate6 & 20 & 12 & 40 & 8 & 26.67 \\
Slight 22 & 73.33 & 15 & 50 & 10 & 33.3 \\
\hline
\end{tabular}

Table IV. Distribution of patients by side effects of therapy.

\begin{tabular}{|lcc|}
\hline Side effects & No. of patients $(\mathrm{n}=6)$ & Percentage $(\mathrm{n}=30)$ \\
General weakness & 1 & $3.33 \%$ \\
Weight gaing & 2 & $6.67 \%$ \\
Acne & 2 & $6.67 \%$ \\
Mild headache & 1 & $3.33 \%$ \\
\hline
\end{tabular}

\section{Discussion}

Among thirty patients of vitligo, most of the male patients $(60 \%)$ and most of the female patients $(73 \%)$ were between 20 to 25 years, having consistent with the study of Amir Habib et al. They observed 80\% males and 20\% females in a cohort Pakistani vitiligo patient. ${ }^{8}$ Majority of patients $(70 \%)$ had been suffering from vitiligo for less than 13 years. In the study by Kim et al in the department of Dermatology, Samsung Cheil Hospital, Sungkyunkwan University School of Medicine, South Korea also observed that the duration of disease were 2 years or less. ${ }^{9}$

In out study, twenty one (70\%) patients had acro-facial and nine patients $(30 \%)$ had generalized vitiligo. The disease was stable in $7(23.3 \%)$ and progressive in $23(76.7 \%)$ cases. The extent of cutaneous involvement was less than $30 \%$ area in $21(70 \%)$ patients, 31 to $50 \%$ area in $8(27 \%)$ patients in the study by Radakovic-Fijan et al. in the department o Dermatology, University of Vienna Medical School, Austria. Of these, 25c had progressive and 4 stable disease. ${ }^{6}$ Amir Habib et al. observed that the disease was stable in $9(30 \%)$ patients and progressive in $21(70 \%)$ patients. They observed that seven $(23 \% 3 \%)$ patients had acrofacial vitiligo and 23 (76.7\%) patients had generalized vitiligo, not similar to our findings. The extent of cutaneous involvement was $1 \%$ to $10 \%$ of body surface area in 19 patients, $11 \%$ to $30 \%$ in 6 patients, $31 \%$ to $50 \% 4$ patients and more than 50 in one patient, similar to our findings. ${ }^{8}$ In our study, response was noticed at first follow-up after 4 weeks and 12 weeks. Response was slight in $73.33 \%$, moderate in $20 \%$, marked in $3.33 \%$ and excellent in $3.33 \%$ of cases after 4 weeks. Response was slight in $33.33 \%$, moderate in $26.67 \%$, marked in $33.33 \%$ and excellent in $6.67 \%$ of cases after 12 weeks. In the study by Radakovic-Fijan et al. after a mean treatment period of 18.2+-5.2 weeks, marked repingmentation occurred in 2 patients $(6.9 \%)$ and moderate or slight repigmentation in 3 patients $(10.3 \%)$ each . No response was noted in 20 patients $(72.4 \%){ }^{6} \mathrm{~A}$ study by Pasricha et al. conducted with forty patients having extensive and/or fast-spreading vitiligo were given $5 \mathrm{mg}$ betamethasone as a single oral dose after breakfast on 2 consecutive days per week. Within 2-4 months of treatment, $80 \%$ of the patients started having spontaneous repigmentation of the existing lesions which progressed with continued treatment. The extent of repigmentation varied in different patients and even in different lesions in the same patient. It was less than $10 \%$ in $14(35 \%)$ patients and almost complete $(>90 \%)$ in three patients. In the study by Amir Habib et al. they observed that regimentation was noted in $14(46.6 \%)$ patients out of 30 patients.

Out of the total 14 patients with repigmentation ,10 (71.43\%) patients had slight, 3 (21.43\%) patients had moderate repigmentation and one $(7.14 \%)$ patient had marked repigmentation. None had excellent repigmentation. No response was observed in the remaining 16 $(53.3 \%)$ patients. $^{8}$ Kim et al were conducted a study in the department of Dematology, Samsung Cheil Hospital, Sungkyunkwan University School of Medicine, Suwon, South Korea. Eighty-one patients with vitiligo were evaluated for 4 months and repigmentation were noted in $40.4 \%$ of patients. ${ }^{9}$ In the present study, $20 \%$ of total patients were seen to be with clinical side effect. Among the side effect- weight gain $(6.67 \%)$ and acne $(6.67 \%)$ was most prevalent, others Include general weakness (3.33\%) and mild headache (3.33\%). Side effects were recorded in 20 patients $(69 \%)$ by Radakovic-Fifan et al. and included weight gain, insomnia, acne, agitation, menstrual disturbance, and hypertrichosis. ${ }^{6}$ The side effects by Pasricha et al included weight gain of 5 and $7 \mathrm{~kg}$ in two patients, mild headache in two patients, transitory general weakness for 2 days after the pulse in two patients, and bad tastes in the mouth in three patients, 23 patients, including six children, had no side effects. ${ }^{7}$ In the study by Amir Habib et al twenty-one (70\%) patients reported one or more side effects such as epigastria burning or pain, bloating, weight gain,. Insomnia, acne and menstrual by the study Kim et al. ${ }^{9}$

Plasma cortisol levels were markedly decreased 48 hours after the second oral mini pulse betamethasone. However, betamethasone induced suppression of endogenous cortisol production was only transitory since plasma cortisol values returned to baseline levels before administration of the next oral mini pulse betamethasone therapy, which is consistent with the study by Amir Habib et al. ${ }^{8}$ The side-effects of treatment were minimal and did not affect the course fo treatment by the study Kim et al. ${ }^{9}$

Plasma cortisol levels were markedly decreased 48 hours after the second oral mini pulse betamethasone. However, 
betamethasone induced suppression of endogenous cortisol production was only transitory since plasma cortisol values returned to baseline levels before administration of the next oral mini pulse betamethasone therapy, which is consistent with the study by Amir Habib et al. ${ }^{8}$ Study by Radakovic-Fajan et al also obsersved that plasma cortisol and corticotrophin values were markedly decreased 24 hours after the second dexamethasone dose, yet returned to baseline values within the off treatment period before the next dexamethasone pulse. ${ }^{6}$

\section{Conclusion}

Oral mini betamethasone seems to be and effective treatment modality with minimal side-effects to arrest the progression of vitiligo and induction fo repigmetnation. It deserves to be tried on a large scal to evaluate its advantages over the currtently available methods of treatment of vitiligo.

\section{References}

1. James WD, Berger TG and Elston DM. Andrew's Disease of the skin-Clinical Dermatology, 10th edition. USA: Sanuders Elsevier; 2006.-p. 860-863.

2. Westerhof W. Vitiligo management update. Skin Therapy Lett 2000; 5(6): 1-2,5.
3. Bleehen SS. Disorders of Skin color. In: Champion RH, Burton JL,Burn DA, Bareathnach SM eds. Rook/Wikinson/ Ebling textbook of Dermatology. London Balckwell Science 1998; 1753-1815.

4. Kovacs So. Vitiligo. J Am Acad Dermatol 1998; 38 : 647-66.

5. Halder RM, Taliaferro SJ. Vitiligo. Wolff K, Goldsmith LA, Katz SI, Gilchrest BA, Paller AS and Leffell DJ. Fitzpatrick's Dermatology in General Medicine. New York. The McGraw-Hill Companies; 2008.p.616-8.

6. Radakovic-Fijan S, Furnsinn-Friedl AM, Honigsmann $\mathrm{H}$, Tanew A Oral dexamethasone pulse treatment for vitiligo. J Am Acad Dermatol 2001; 44(5): 814-7.

7. Pasircha JS, Khaitan BK. Oral mini-pulse therapy with betamethasone in vitiligo patients having extensive or fast spreading disease. Int J Dermatol 1993; 32(10): 753-7.

8. Aamir Habib, Zafar Iqbal Sheikh, Qamar ud Din Khan, Simeen Ber Rahman. Efficacy and safety of oral dexamethasone pulse treatment for vitiligo. Pakistan Armed Forces Medical Journal 2006 June; 2:1-6.

9. Kim SM, Lee HS, Hann SK. The efficacy of low-dose oral corticosteroids in the treatment of vitiligo patients. Int J Dermatol. 199 Jul; 38(7)L546-50. 